\title{
Balancing our biases
}

\author{
Jay D. Pal, MD, PhD, and Nahush A. Mokadam, MD
}

\footnotetext{
From the Division of Cardiothoracic Surgery, Department of Surgery, University of Washington Medical Center, Seattle, Wash.

Disclosures: J.D.P. has a consulting relationship with Thoratec Corporation (Pleasanton, Calif) and HeartWare International Inc (Framingham, Mass). N.A.M. has a consulting relationship with Thoratec Corporation (Pleasanton, Calif), HeartWare International, Inc (Framingham, Mass), and St Jude Medical Center (Fullerton, Calif).

Received for publication Oct 11, 2015; accepted for publication Oct 17, 2015.

Address for reprints: Nahush A. Mokadam, MD, Division of Cardiothoracic Surgery, Department of Surgery, University of Washington Medical Center, Box 356310, 1959 NE Pacific St, Seattle, WA 98195 (E-mail: mokadamn@uw.edu).

J Thorac Cardiovasc Surg 2016;151:211-2

0022-5223/\$36.00

Copyright (c) 2016 by The American Association for Thoracic Surgery

http://dx.doi.org/10.1016/j.jtcvs.2015.10.053
}

We live our personal and professional lives full of bias. By their very nature, biases are irrational and difficult to overcome. They can result from lifelong influences, anecdotal experiences, or even scientific discovery. As clinicians, we have a responsibility to properly weigh the sources of our opinions, be critical, re-evaluate, and remain open minded.

The biases in the management of aortic insufficiency during left ventricular assist device (LVAD) implantation have evolved over time: Early in our experience, procedures requiring cardioplegic arrest of the heart were associated with increased mortality. ${ }^{1}$ In addition, the duration of mechanical circulatory support was relatively short in most patients. These factors led to relative resistance to adding procedures during LVAD implantation. More recently, we have learned that aortic insufficiency does increase during periods of mechanical support, and that aortic valve procedures can be performed safely during LVAD implantation. ${ }^{2,3}$ As support time increases, the progression of aortic insufficiency may become more relevant. However, the incidence of concomitant aortic valve procedures has remained low, despite growing evidence that outcomes with progressive aortic insufficiency are compromised.

A recent Interagency Registry for Mechanically Assisted Circulatory Support database analysis supports the position that aortic valve procedures at the time of LVAD implantation increase perioperative risk. ${ }^{4}$ The bias introduced here is of course one of selection-the presence of aortic valve insufficiency may represent a sicker cohort. Nonetheless, we cannot ignore data that support our preconceived ideas that aortic valve procedures add unnecessary risk. So how are we to properly evaluate the importance of addressing aortic insufficiency at the time of LVAD implantation?

The answers, of course, come from good data. In this issue, Fukuhara and colleagues ${ }^{5}$ present their center's outcome data on patients who undergo concomitant central aortic valve closure (CAVC with Park's stitch) during implantation of a continuous-flow LVAD. The authors

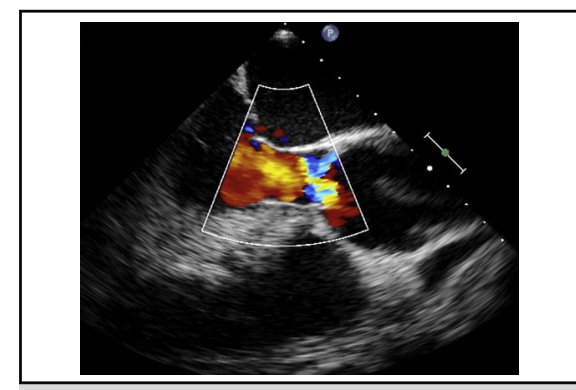

Parasternal long-axis view of the aortic valve, depicting aortic insufficiency.

\section{Central Message}

The bias toward viewing aortic valve closure as harmful has been challenged; the procedure may be beneficial for patients who have LVADs.

See Article page 201.

See Editorials page 10 and 13.

describe their center's extensive experience with LVAD implantation with a granular level of detail that is not possible with large database analyses. They report that CAVC can be performed safely, and more importantly, can positively affect perioperative and long-term outcomes.

Although their report ${ }^{5}$ demonstrates several perioperative impacts of additional procedures (additional cardiopulmonary bypass and myocardial ischemic time, additional transfusions, and increased inotropic requirements), operative mortality is unchanged. Furthermore, they demonstrate that CAVC can decrease the likelihood of development of late aortic insufficiency, especially in older destination therapy patients. This latter finding is particularly important, because these patients can redevelop heart failure symptoms for which few therapeutic options are available.

To be fair, we must question whether a type II error in the determination of overall survival has occurred, owing to the relatively few CAVC procedures performed in the overall cohort. To the casual eye, the survival curves do seem to diverge, but statistical analysis does not show a difference. Further, although the development of aortic insufficiency is limited by CAVC, whether the development of aortic insufficiency portends negative clinical outcomes in these patients is unclear from this report. Nonetheless, this report challenges the concept that aortic valve procedures are harmful; in fact, they may be beneficial in properly selected patients.

So, how are we to balance these seemingly contradictory findings? Are we to lean on reports that support evidence of 
harm from aortic valve procedures? Or will reports such as this one prompt us to challenge existing dogma? As a specialty, we will continue to debate this topic, and this report provides balance to a large database series. A concept we teach our medical students is relevant here: If given more than one answer for a problem, by different individuals, then the data are likely insufficient to resolve the issue; the divergent opinions are often the result of bias.

\section{References}

1. Pal J, Klodell C, John R, Pagani FD, Rogers JG, Farrar DJ, et al. Low operative mortality with implantation of a continuous-flow left ventricular assist device and impact of concurrent cardiac procedures. Circulation. 2009;120(11 Suppl): S215-9.

2. Milano C, Pagani FD, Slaughter MS, Pham DT, Hathaway DR, Jacoski MV, et al. Clinical outcomes after implantation of a centrifugal flow left ventricular assist device and concurrent cardiac valve procedures. Circulation. 2014;130(11 Suppl 1):S3-11.

3. Cowger J, Pagani FD, Haft JW, Romano MA, Aaronson KD, Kolias TJ. The development of aortic insufficiency in left ventricular assist device-supported patients. Circ Heart Fail. 2010;3:668-74.

4. Robertson JO, Naftel DC, Myers SL, Prasad S, Mertz GD, Itoh A, et al. Concomitant aortic valve procedures in patients undergoing implantation of continuous-flow left ventricular assist devices: an INTERMACS database analysis. J Heart Lung Transplant. 2015;34:797-805.

5. Fukuhara S, Takeda K, Chiuzan C, Han J, Polanco AR, Yuzefpolskaya M, et al. Concomitant aortic valve repair with continuous-flow left ventricular assist devices: results and implications. J Thorac Cardiovasc Surg. 2016;151:201-10.e2. 